Itinéraires Itinéraires

Littérature, textes, cultures

\title{
Modernité, représentation de l'histoire et présentisme dans le théâtre shakespearien
}

Jean-Christophe Mayer

\section{(2) OpenEdition}

\section{Journals}

Édition électronique

URL : http://journals.openedition.org/itineraires/1730

DOI : 10.4000/itineraires. 1730

ISSN : 2427-920X

Éditeur

Pléiade

\section{Édition imprimée}

Date de publication : 1 décembre 2010

Pagination : 101-115

ISBN : 978-2-296-13183-5

ISSN : 2100-1340

Référence électronique

Jean-Christophe Mayer, « Modernité, représentation de l'histoire et présentisme dans le théâtre shakespearien », Itinéraires [En ligne], 2010-4 | 2010, mis en ligne le 01 décembre 2010, consulté le 30 avril 2019. URL : http://journals.openedition.org/itineraires/1730; DOI : 10.4000/itineraires. 1730

\section{(C) $(\oplus \Theta$}

Itinéraires est mis à disposition selon les termes de la licence Creative Commons Attribution - Pas d'Utilisation Commerciale - Pas de Modification 4.0 International. 


\title{
Modernité, représentation de l'histoire et présentisme dans le théâtre shakespearien
}

\begin{abstract}
This article explores the links between modernity and presentism in the work of Shakespeare and of some of his fellow Elizabethans, as well as in the writings of the most avant-garde of contemporary literary critics. It stresses the paradoxical nature of the notion of "modernity", which questions its own values endlessly. The notion invites us to investigate the complex dialectic of the old and the new both in Shakespeare's time and in our own. The conclusion reached is that the current turn to presentism in Shakespearean criticism is seriously misguided.
\end{abstract}

Keywords : Shakespeare, modernity, presentism, history, representations

Mots clés : Shakespeare, modernité, présentisme, histoire, représentation

\section{Définitions et usages de la modernité}

Si la modernité faisait bien partie du lexique des Anglais au début du XVII ${ }^{\mathrm{e}}$ siècle, comme l'atteste l'entrée « modernity » de l'Oxford English Dictionary, il faut reconnaître que l'usage de ce substantif demeurait encore très limité, mais qu'il pouvait déjà désigner la nature actuelle d'un style ou d'une esthétique. L'adjectif « moderne » (« modern ») était quant à lui bien plus répandu à l'époque de Shakespeare. Il désignait (toujours selon l'Oxford English Dictionary) ce qui était actuel, contemporain, ou encore ce qui se distinguait d'autres époques. Enfin, il pouvait faire référence, chez Shakespeare notamment, à l'ordinaire, le commun, ou le quotidien ${ }^{1}$.

Comme on peut le constater, ces usages témoignent tout à la fois du développement d'une conscience historique (impliquant en particulier une

1. C'est le sens le plus répandu des neuf occurrences de l'adjectif que l'on trouve réparties dans des œuvres comme le sonnet 83, Roméo et Juliette, Le Roi Jean, Comme il vous plaira, Tout est bien qui finit bien, Othello, Macbeth, Antoine et Cléopâtre. 
division de l'histoire en périodes) et d'un goût croissant pour l'éphémère, l'actuel et la nouveauté. Pour le critique littéraire ou pour l'historien, les notions de « moderne » ou de « modernité » sous-entendent l'élaboration d'une conscience historique potentiellement ambivalente, ainsi que le développement d'une perception esthétique paradoxale. Ainsi la Renaissance est-elle une période qui aime à se comparer à l'Antiquité, dont elle prétend imiter les valeurs tout en s'en détachant (le Moyen Âge pouvant être considéré en partie comme une notion destinée à mettre en valeur le $\mathrm{XVI}^{\mathrm{e}}$ siècle, à marquer sa différence ${ }^{2}$ ). Pour le critique littéraire, ou l'historien de l'art, cette différence est fondamentalement paradoxale, car le mot «modernité » présente effectivement « ce paradoxe de démentir à l'évidence à tout instant, par sa récurrence historique, la prétention qu'il affirme ${ }^{3} »$.

Chaque époque a donc potentiellement sa conception du « moderne », la modernité étant un concept qui se définit constamment par opposition à lui-même. Ce concept en dit long en fait sur le rapport des êtres humains au passé, et, d'un point de vue esthétique et critique, sur la dialectique de l'ancien et du nouveau qui se dissimula derrière la querelle des anciens et des modernes pendant les $\mathrm{XVII}^{\mathrm{e}}$ et $\mathrm{XVIII}{ }^{\mathrm{e}}$ siècles, puis derrière celle qui opposa romantisme et classicisme au XIX ${ }^{\mathrm{e}}$ siècle. C'est d'ailleurs du XIX ${ }^{\mathrm{e}}$ siècle que nous vient l'acception actuelle de la notion de modernité. Dans « Le peintre de la vie moderne » (1868), Baudelaire donna ses lettres de noblesse à la modernité en décrivant le geste sans cesse répété de l'artiste qui extrait du présent ce qui est digne d'être préservé. Il s'agit pour lui de rechercher le " poétique dans l'historique, de tirer l'éternel du transitoire 4 ». La modernité, c'est la recherche de la beauté dans le transitoire et le fugitif. « En un mot ", écrit Baudelaire, "pour que toute modernité soit digne de devenir antiquité, il faut que la beauté mystérieuse que la vie humaine y met involontairement en ait été extraite » (p. 517). Il ne s'agit pas de privilégier l'ancien sur le nouveau, ni même de faire du nouveau la référence ultime. Il est plutôt question de laisser la dialectique de l'ancien et du nouveau opérer pleinement afin qu'elle produise ses fruits. L'opposition modernité/ Antiquité reste stérile tant que le croisement de l'ancien et du nouveau au cours des présents successifs n'est pas étudié. Car, comme l'écrit Paul Ricœur, « si le présent historique peut prétendre se penser lui-même, ce ne peut être que comme point nodal de l'universel et de l'historique ${ }^{5} »$.

2. «L'entente entre antique et moderne se fait sur le dos du Moyen Âge » (Jacques Le Goff, Histoire et mémoire, Paris, Gallimard, 1988, p. 71).

3. Hans Robert Jauss, "La Modernité" dans la tradition littéraire et la conscience d'aujourd'hui », dans Pour une esthétique de la réception, trad. Claude Maillard, préf. Jean Starobinski, Paris, Gallimard, 1978, p. 173.

4. Charles Baudelaire, « Le peintre de la vie moderne », dans Henri Lemaitre (éd.), Curiosités esthétiques [1868], Paris, Bordas, 1990, p. 515. Les références suivantes seront données dans le texte.

5. Paul Ricœur, La Mémoire, l'histoire, l'oubli, Paris, Seuil, 2000, p. 411. 
Mais qu'en est-il de la période shakespearienne? Comment comprendre la dialectique de l'antique et du nouveau dont témoignent nombre d'écrits de l'époque? La modernité et la nouveauté faisaient le plus souvent l'objet d'une certaine suspicion, même si elles n'étaient pas vues systématiquement sous un jour négatif. Un regard rétrospectif n'impliquait pas nécessairement une déférence nostalgique vis-à-vis de l'Antiquité, comme l'indiquait Henry Peacham dans un traité publié en 1622 :

\footnotetext{
Mais quand nous regardons vers l'Antiquité, n'oublions pas nos temps modernes et plus tardifs (ne croyons pas que la nature aurait produit sa quintessence dans le passé pour ne nous laisser que la lie); notre époque a produit des esprits aussi féconds que dans les temps passés, et qui plus est dans notre pays, la Grande-Bretagne.
}

Pour Peacham, la modernité n'est pas synonyme de déchéance, mais de créativité (cf. " esprits aussi féconds $\left.{ }^{6} »\right)$. On comprend que l'apologie de la modernité était nécessaire pour ceux qui, à l'instar de Peacham, avaient à cœur la défense de l'identité nationale britannique (cf. « notre pays, la Grande-Bretagne »).

D'autres auteurs préféraient s'inscrire dans une lignée plus illustre, le lien avec l'Antiquité devenant un facteur valorisant pour leurs travaux. Dans une préface adressée au comte de Somerset, George Chapman défend la traduction qu'il a publiée de l'Odyssée d'Homère :

\footnotetext{
Pourquoi un mauvais chroniqueur, narrant dans toute sa nudité le récit véridique d'un lord-maire (qui n'excèdera sans doute pas une année) estil mieux considéré par nos philosophes modernes qu'Homère drapant la nudité d'Ulysse dans le manteau éternel de la fiction?
}

Il est aisé de percevoir ici un certain mépris intellectuel à l'encontre de ceux qui étaient friands des chroniques d'un passé civique récent et éphémère ${ }^{7}$.

Ben Jonson lui aussi ironisait parfois sur l'appropriation d'auteurs contemporains illustres comme le poète et dramaturge italien Giovanni Battista Guarini, ou même Montaigne, dont la prétendue "modernité » était transformée en distraction pour des contemporains (notamment des courtisans) soucieux de s'inspirer des dernières tendances de la mode littéraire. Dans Volpone, la très anglaise Lady Jacasse («Lady Politic

6. Henry Peacham, The Compleat Gentleman. Fashioning Him Absolute, in the Most Necessarie and Commendable Qualities Concerning Minde or Bodie, That May Be Required in a Noble Gentleman, London, Printed [by G. Wood] for Francis Constable, and are to be sold at his shop in Pauls Church-yard, at the signe of the Crane, 1627, p. 91. Source : Literature Online. Ma traduction.

7. Homer's Odysses. Translated According to Ye Greeke by. Geo : Chapman, Imprinted at London : By Rich : Field, for Nathaniell Butter, 1614, sig. A4v․ STC : 13636. Ma traduction. 
Would-be », dans l'original) confie au personnage éponyme italien son goût immodéré pour la « modernité » de ces auteurs :

Tous nos écrivains anglais,

J'entends tous ceux qui sont à l'aise en italien,

N'hésitent pas à piller cet auteur;

Au moins autant que Montaigne.

Sa veine est si moderne, si facile, si adaptée

À notre temps, si plaisante à l'oreille de la Cour ${ }^{8}$.

Cette veine dite « moderne » s'adapte facilement, selon Lady Jacasse, au goût de ses contemporains, cette adaptation laissant chez le spectateur ou le lecteur une impression de superficialité. Sous certaines de ses formes, la modernité se révèle parfois vide de sens. Cette vacuité peut produire de l'humour, mais elle peut également susciter une anxiété de nature métaphysique. C'est le cas lorsque le vieux Lord Lafeu s'étonne quelque peu que, selon les philosophes (mais aussi certainement selon certains protestants), l'on ne doive plus croire aux miracles, que les événements tout simples («modern») et familiers sont dépourvus d'une causalité divine directe :

On dit qu'il n'y a plus de miracles; et nous avons des philosophes qui déclarent toutes simples [en anglais : «modern»] et toutes ordinaires les choses surnaturelles et inexplicables. Voilà ce qui fait que nous traitons de puérilités les plus redoutables prodiges, en nous retranchant dans une science prétendue, au lieu de nous résigner à une ignorante terreur ${ }^{9}$.

Pourtant, cette connaissance nouvelle et cette confiance envers les dogmes modernes n'aboutiront peut-être qu'à des illusions (cf. «science prétendue ») nous empêchant d'éprouver un respect teinté d'effroi devant les mystères métaphysiques. L'on sent ici poindre un doute et une hésitation visà-vis d'une modernité dont les contours ne sont pas clairement saisissables.

\section{Regards critiques sur la modernité : Shakespeare et les genres de l'éphémère}

L'un des genres qui, à la Renaissance, accorde une large place à la dialectique entre l'ancien et le nouveau est sans doute la pièce historique, et notamment (mais pas exclusivement) le drame historique shakespearien. Profitant de toute une production d'écrits (pamphlets et courts traités écrits en réponse à une actualité récente, ballades, ou encore lettres de

8. Ben Jonson, Volpone ou le Renard, trad. Michèle Willems, dans Line Cottegnies, François Laroque et Jean-Marie Maguin (dir.), Théâtre élisabéthain, 2 vols., Paris, Gallimard, coll. « Bibliothèque de la Pléiade », 2009, vol. 2, p. 81 (Acte III, scène IV). La pièce fut jouée aux alentours de 1605-1606 et sa première édition date de 1607 .

9. William Shakespeare, Tout est bien qui finit bien, trad. François-Victor Hugo, dans Henri Fluchère (dir.), Euvres complètes, 2 vols., Paris, Gallimard, coll. " Bibliothèque de la Pléiade », 1989, vol. 2, p. 259 (Acte II, scène III). 
nouvelles) qui déplaçaient progressivement les anciens repères imposés par la chronique, le drame historique allait lui aussi opérer un redécoupage du temps. Dans son rapport à la temporalité, ce théâtre laissait parfois entrevoir des doutes et des hésitations. En effet, le théâtre historique est un genre intermédiaire qui joue sur plusieurs tableaux : celui de l'histoire, de la chronique, mais aussi celui du présent. La pièce historique se situe au cour des batailles que se livrèrent les genres de l'éphémère à la fin de la période élisabéthaine : éphémère par essence, souvent réactive, et parfois influencée par l'actualité, la pièce historique, dans son travail de reconstruction du passé, joue sans cesse sur la frontière entre le passé et le présent, une frontière mouvante dont les contours sont néanmoins ébauchés à chaque représentation.

Ainsi, le théâtre de Shakespeare propose un regard malicieux, parfois critique, sur un genre avec lequel il pouvait être en rivalité, tant sur «le marché du passé » que sur celui du présent ${ }^{10}$. La ballade est un genre de l'éphémère, qui a prospéré en s'appuyant en partie sur les chroniques, mais qui prétend également répondre aux caprices de l'actualité. Dans Henry IV, deuxième partie, Falstaff, qui pourtant ne fut pas un modèle de bravoure, souhaite que ses " exploits » sur le champ de bataille soient inscrits ( enregistrés ») dans un livre pour la postérité; Shakespeare lui fait promettre, sinon, qu'il fera imprimer une ballade portant son nom, accompagnée d'un portrait de lui-même : "j'insiste auprès de Votre Grâce pour que ce soit enregistré avec les autres hauts faits du jour, ou, par Dieu, je le mettrai alors dans une ballade à part, avec mon propre portrait audessus, Coleville me baisant le pied ${ }^{11}$. \ Par le biais de la caricature, la pièce historique shakespearienne souligne que son personnage se situe bien à la croisée de deux genres, celui de la chronique et celui de la ballade ${ }^{12}$.

10. Les chroniques, les traités historiques, les courtes histoires, de même que les ballades consacrées à des événements du passé représentaient à l'époque un marché qui, sans égaler celui des livres religieux, n'était pas négligeable et allait continuer de se développer pendant la période moderne. Voir, à ce sujet, le chapitre intitulé « Marketing History » dans D. R. Woolf, Reading History in Early Modern England, Cambridge, Cambridge University Press, 2000, p. 255-317.

11. William Shakespeare, 2 Henry IV, trad. Michel Grivelet, dans Michel Grivelet et Gilles Monsarrat (dir.), Histoires, éd. bilingue, Paris, R. Laffont, 1997, p. 693 (Acte IV, scène II).

12. On peut remarquer en passant que Shakespeare fut obligé de gommer par la suite les interférences avec l'actualité produites par ce personnage. Le dramaturge transforma, en effet, le personnage de Oldcastle (Henry IV, première partie) en Falstaff dans Henry IV, deuxième partie pour éviter de froisser la famille des Cobham, les descendants du martyr protestant Sir John Oldcastle, Lord Cobham. Sur le genre de la ballade historique, on pourra se reporter à : C. H. Firth, «The Ballad History of the Reigns of the Later Tudors », Transactions of the Royal Historical Society, $3^{\mathrm{e}}$ série, ${ }^{\circ}$ 3, 1909, p. 51-124; C. H. Firth, « The Ballad History of the Reign of James I », Transactions of the Royal Historical Society, $3^{\mathrm{e}}$ série, $\mathrm{n}^{\circ}$ 5, 1911, p. 21-61. Sur la ballade comme contre-histoire, voir Daniel Woolf, The Social Circulation of the Past : English Historical Culture 1500-1730, Oxford, Oxford University Press, 2003, p. 322-340 passim. 
Les affinités de la ballade et du théâtre sont évidentes, dans la mesure où la ballade, comme le théâtre, peut proposer une histoire qui n'est pas nécessairement le reflet des chroniques, et en ce sens elle peut, comme le théâtre quelquefois, laisser entrevoir la possibilité d'une contre-histoire ${ }^{13}$.

Dans Henry IV, deuxième partie, le théâtre jette un regard amusé sur la fabrication d'une histoire frauduleuse, d'un passé de pacotille, d'une réputation fictive. Mais l'œuvre de Shakespeare qui s'intéresse de plus près à la ballade est certainement Le Conte d'hiver (1609-1611). Cette pièce témoigne de l'incroyable succès du genre dans une scène où Autolycus, un colporteur canaille, tente de vendre des ballades à de naïfs habitants des campagnes. Bien que leur sujet soit souvent fabuleux, Autolycus prétend que ces ballades relatent des faits récents et véridiques :

Le Clown. - Qu'as-tu ici? des ballades?

Mopsa. - Je vous en prie, achetez-en : j'adore ça, une ballade imprimée, qui raconte une vie, car alors on est sûr que c'est véritable.

Autolycus. - En voici une sur un air très lamentable: où l'on voit comment la femme d'un usurier accoucha d'un seul coup de vingt sacs d'argent, et comme quoi elle eut des envies de manger des têtes de vipères et des crapauds en carbonnade.

Mopsa. - C'est vrai, vous croyez?

Autolycus. - Parfaitement vrai; et il n'y a pas plus d'un mois que c'est arrivé $^{14}$.

On devine comment Shakespeare eut l'idée d'écrire cette scène pour son Conte d'hiver. En contemplant d'autres genres qui se situaient eux aussi à la frontière ténue entre le véridique, le merveilleux et l'illusion totale (ou la supercherie), Shakespeare tentait également de mieux définir la place de sa propre pratique théâtrale. Comme dans le masque de Jonson, Nouvelles du Nouveau Monde découvert sur la lune (Newes from the New World Discover'd in the Moone, 1620), on trouve dans cette scène une critique du commerce qui est fait de l'actualité, commerce qui devient plus explicitement la cible d'un dramaturge comme Jonson ${ }^{15}$.

13. Ces affinités étaient d'autant plus grandes que le théâtre de Shakespeare inspira à son tour les auteurs de ballades. Des pièces comme Le Roi Lear, Titus Andronicus, La Mégère apprivoisée, ou Le Marchand de Venise, firent leur apparition plus tard sous forme de ballades. Voir, sur cette fascinante circulation culturelle, Thomas Percy, Reliques of Ancient English Poetry, 3 vol., Londres, Edward Moxon, 1846, vol. 1, p. 166-172, 176-181, 182-188, 189192; Bruce R. Smith, «Shakespeare's Residuals : The Circulation of Ballads in Cultural Memory », dans Stuart Gillespie et Neil Rhodes (eds.), Shakespeare and Elizabethan Popular Culture, Londres, Arden Shakespeare, 2006, p. 193-217.

14. William Shakespeare, Le Conte d'hiver, trad. Suzanne Bing et Jacques Copeau, dans Henri Fluchère (dir.), Euvres complètes, vol. 2, p. 1441 (Acte IV, scène IV).

15. Ben Jonson, Newes from the New World Discover'd in the Moone, dans C. H. Herford Percy et Evelyn Simpson (eds.), Ben Jonson, Oxford, Clarendon Press, 1952, vol. 7, p. 511-525. Ma traduction. Toutes les références subséquentes seront données dans le texte. 
Dans Nouvelles du Nouveau Monde découvert sur la lune, Jonson met en scène la rencontre de deux hérauts, d'un chroniqueur, d'un imprimeur et d'un auteur de nouvelles épistolaires. Le dramaturge montre comment chacun construit, invente le présent en fonction des différents médias employés. Il souligne aussi que l'actualité est désormais un objet commercial comme un autre, qui se vend et s'achète, quand bien même les nouvelles seraient factices. La seule différence entre l'imprimeur et le colporteur de nouvelles manuscrites tient au fait que chacun croit dur comme fer que son support est le mieux adapté à la diffusion de l'actualité : pour le colporteur, les nouvelles ne sont plus fraîches si elles sont imprimées (p. 515, 1. 57-59); pour l'imprimeur, au contraire, celles-ci deviennent justement des nouvelles parce que le support imprimé exerce un pouvoir de persuasion sur les personnes (p. 515, 1. 61-63). Le cynisme de l'imprimeur montre qu'il est engagé dans une production de fausses nouvelles sur mesure destinées aux masses (p. 515, 1. 52-54), et que cette production de l'actualité, sur un mode préindustriel, tourne en boucle, puisque notre imprimeur avoue recycler l'actualité, faire du neuf avec du vieux en modifiant les dates : " Tous les dix ans (notre époque étant oublieuse) je réimprime les nouvelles en changeant les dates, et celles-ci font encore merveille» (p. 515, 1. 65-67). Dans une autre pièce au titre éloquent, The Staple of Newes (1625), c'est-à-dire La Boutique aux Nouvelles, Jonson reprend ces mêmes thèmes en insistant sur les aspects les plus critiquables de ce commerce du présent, notamment lorsque la fameuse fabrique de nouvelles, dont il est question dans cette pièce, se met à produire des rumeurs, et se révèle des plus manipulatrices, en particulier envers ceux dont l'esprit critique est le moins aiguisé ${ }^{16}$.

Malgré son titre volontairement racoleur, La Boutique aux Nouvelles de Jonson n'a aucune véritable nouvelle à annoncer, même si elle raconte quel commerce peut être fait de l'actualité. Bien qu'il sache que certains sont venus voir sa pièce pensant grignoter en écoutant des potins (« les plus vulgaires / Des casseurs de noisettes qui viennent uniquement pour voir », p. 6), Jonson propose à son spectateur (notamment à son public de la Cour) une analyse critique et satirique du goût immodéré de ses contemporains pour le présent :

\footnotetext{
Même si, Messieurs, notre titre parle de « Nouvelles »,

Nous n'avons pas l'intention de vous en donner;

Des folies courantes nous allons plutôt vous montrer,

Et si celles-ci ne contiennent rien de vrai, l'innocente Muse, elle,

Fait en sorte que l'imagination puisse les dire,

Ou que la poésie puisse sans honte les décrire. (Ibid.)
}

16. Voir, en particulier : Ben Jonson, The Staple of Newes, dans The workes of Benjamin Jonson, Londres, Richard Meighen 1640, p. 41 (Acte III, scène II). Source : Literature Online. Ma traduction. Références subséquentes données dans le texte. 


\section{Les écueils du présentisme}

Shakespeare et Jonson avaient de toute évidence compris que le présent commençait à revêtir une importance toute particulière pour leurs contemporains, qui allaient, au fil des ans, devenir d'avides consommateurs d'actualité. Il est clair également que ces dramaturges avaient bien saisi les enjeux commerciaux de ce goût prononcé pour le présent.

Derrière ce goût avéré des contemporains de Shakespeare et de Jonson pour «l'actuel », il serait tentant de voir l'émergence d'un présentisme, c'est-à-dire de l'opinion qu'une connaissance absolue du passé étant impossible, toute reconstruction historique n'est en fait que le reflet des préoccupations du présent, qui devient par là même le seul objet d'intérêt digne de ce nom, car lui seul nous est accessible. Il peut paraître saugrenu d'appliquer cette idée à une époque, la Renaissance, dont on sait qu'elle était tournée en partie vers le passé et engagée dans la renovatio des exampla classiques. C'est pourtant ce qu'a tenté de faire récemment une frange non négligeable de la critique shakespearienne ${ }^{17}$. Du point de vue de l'histoire de la critique, l'idée de présentisme n'est évidemment pas une nouveauté. On peut penser en effet à la mise en garde de Nietzsche visant à prévenir les excès d'une science historique dont les travaux stériles ne seraient d'aucun secours pour le présent : «le passé doit être oublié sous peine de devenir le fossoyeur du présent ${ }^{18}$. " Derrière le présentisme d'une partie de la critique shakespearienne, on devine encore le spectre de Foucault, pour qui toute position de surplomb est intenable, l'histoire totale étant impossible :

Cette histoire des historiens se donne un point d'appui hors du temps; elle prétend tout juger selon une objectivité d'apocalypse; mais c'est qu'elle a supposé une vérité éternelle, une âme qui ne meurt pas, une conscience toujours identique à soi ${ }^{19}$.

17. On peut citer, à titre d'exemples, les travaux suivants : Terence Hawkes, Shakespeare in the Present, Londres et New York, Routledge, 2002; Ewan Fernie, « Shakespeare and the Prospect of Presentism », Shakespeare Survey, $n^{\circ}$ 58, 2005, p. 169-184; Hugh Grady et Terence Hawkes (eds.), Presentist Shakespeares, Londres et New York, Routledge, 2007. Toutes les références subséquentes à ces ouvrages seront données dans le texte.

18. Friedrich Nietzsche, Seconde Considération Intempestive, De l'utilité et de l'inconvénient des études historiques pour la vie [1874], trad. Henri Albert, intro. Pierre-Yves Bourdil, Paris, Flammarion, 1988, p. 78. Ce sont bien les excès de l'histoire qui sont visés par Nietzsche, et non pas l'histoire elle-même à qui le philosophe reconnaît bien évidemment un rôle crucial : «La parole du passé est toujours parole d'oracle. Vous ne l'entendrez que si vous êtes les constructeurs de l'avenir et les interprètes du présent » (ibid., p. 131); ou encore, «L'excès des études historiques a affaibli la force plastique de la vie, en sorte que celle-ci ne sait plus se servir du passé comme d'une nourriture substantielle » (ibid., p. 174).

19. Michel Foucault, « Nietzsche, la Généalogie, l'Histoire », dans Suzanne Bachelard, Georges Canguilhem et al. (dir.), Hommage à Jean Hyppolite, Paris, PUF, 1971, p. 145-151; p. 159. 
De ce fait, l'histoire devient l'histoire des subjectivités émanant d'un présent qui ne peut faire autre chose que de construire des passés à son image. Foucault transforme donc la part de subjectivisme inhérente à toute réflexion humaine en relativisme total, condamnant les sciences humaines à sombrer dans une sorte de nombrilisme.

Dans Shakespeare and the Present, Terence Hawkes se fait l'apôtre d'un postmodernisme totalement décomplexé : le présentisme tel qu'il le pratique ne s'intéresse « qu'au présent dans sa matérialité qui est le point de départ de ses interrogations »; il s'agit pour lui de «jouer cartes sur table » et d'appeler chacun à faire preuve « d'une conscience accrue de ses pratiques critiques » (p. 22). Ewan Fernie, quant à lui, surenchérit dans un article récent (« Shakespeare and the prospect of presentism »), proposant une perspective que l'on ne peut qualifier que de radicale. Avec des accents nietzschéens, mais sans les précautions du philosophe, il déclare

qu'il y a une raison évidente (et du reste irréfutable) pour laquelle il faut préférer le présent : celui-ci se déroule maintenant. Le présent, c'est là où nous vivons et nous pouvons toujours intervenir pour le changer. Et c'est précisément pourquoi il a bien plus d'importance que le passé. (p. 178)

Frôlant le truisme, Fernie semble aussi instrumentaliser Shakespeare à des fins politiques, confondant critique littéraire et militantisme. Mais le plus inquiétant réside surtout dans le fait que Presentist Shakespeares (2007), un ouvrage collectif sous la direction de Hugh Grady et de Terence Hawkes, qui était censé montrer le potentiel du nouveau mouvement critique se réclamant du " présentisme », ne consacre pas une seule page à la définition de ce qu'il faut entendre par « présent ». Partant de la (fausse) croyance que le présent est plus saisissable que le passé parce qu'il est plus proche de nous, ces critiques courent le risque de transformer la critique littéraire en mauvais journalisme.

Vouloir fonder une pratique littéraire sur un présent présumé plus accessible, c'est se condamner à devenir la victime d'un leurre. En effet, le présent n'est-il pas tout aussi mystérieux et insondable que le passé? La compréhension d'un présent même proche implique nécessairement un travail de reconstruction qui est semblable à celui pratiqué par les historiens lorsqu'ils se penchent sur le passé. Le présent n'est pas plus donné que le passé. Comme le faisait déjà remarquer Puttenham dans L'Art de la poésie anglaise (1589), c'est bien la vitesse à laquelle se déroule le présent qui le rend insaisissable immédiatement :

Le temps et les événements présents passent si vite que nous n'avons presque pas le loisir de nous pencher sur eux, et encore moins de les connaître ou de les examiner en détail ${ }^{20}$.

20. George Puttenham, The Arte of Englishe Poesie, Londres, Richard Field, 1589; Menston, Scolar Press, 1968, p. 31-32. Ma traduction. Pour une critique du présentisme, voir aussi : 
En termes modernes, on pourrait dire, avec Paul Veyne, que « la conscience spontanée ne possède pas de notion d'histoire, qui exige une élaboration intellectuelle ${ }^{21} \gg$. Or il semblerait que l'on ait inversé les anciennes prétentions d'une critique qui se voulait totalisante et objective, en proclamant l'avènement d'une ère nouvelle : celle d'une méthode d'analyse qui place sa subjectivité au cœur de sa pratique et fait de son temps (le présent) l'essentiel de ses préoccupations. Il y a là un retournement qui a toutes les apparences d'une réaction de rejet d'une ancienne méthode; et pourtant, ce retournement n'est qu'apparence, car le présent ne peut pas plus se penser lui-même qu'il ne peut prétendre penser totalement le passé. Comme le souligne justement Paul Ricœur, « la singularité historique se pensant elle-même suscite une aporie symétrique de celle de la totalité historique se sachant absolument ${ }^{22} \gg$. Au sens littéral du terme, une histoire du présent est impossible ${ }^{23}$. Toute méthode critique visant à parler du présent est forcément une pratique a posteriori qui implique un travail de reconstruction, sujet à la même part de subjectivité que l'étude du passé, et dépendant dans les deux cas (lorsqu'il est pratiqué avec discernement) des mêmes méthodes scientifiques (analyse critique des sources, recoupement des informations, etc.). Nous ne voyons donc pas pourquoi l'on abandonnerait l'étude du passé au nom d'un relativisme qui s'applique également à l'analyse du présent.

Le présentisme est sans doute l'enfant d'un $\mathrm{XX}^{\mathrm{e}}$ siècle en proie au désarroi, désorienté par des transformations sociales, culturelles et politiques, qui se sont succédé à un rythme prodigieux. On peut penser à cet égard à « l'Hamlet européen » que décrit Paul Valéry dans une méditation présentiste qui date de 1919. Hamlet est pour Valéry la figure de l'homme moderne «accablé sous le poids des découvertes, des connaissances, incapable de se reprendre à cette activité illimitée. Il songe à l'ennui de recommencer le passé, à la folie de vouloir innover toujours. Il chancelle

Robert D. Hume, Reconstructing Contexts : The Aims and Principles of Archaeo-Historicism, Oxford, Oxford University Press, 1999, p. 181; Robin Headlam Wells, « Historicism and "Presentism" in Early Modern Studies ", The Cambridge Quarterly, n 29/1, 2000, p. 37-60; Robin Headlam Wells, «Afterword : historicism and "presentism" », dans Shakespeare on Masculinity, Cambridge, Cambridge University Press, 2000, p. 207-218.

21. "L'histoire est une notion livresque et non un existential [sic]; elle est l'organisation par l'intelligence de données qui se rapportent à une temporalité qui n'est pas celle du Dasein » (Paul Veyne, Comment on écrit l'histoire [1971], Paris, Seuil, 1978, p. 101).

22. Paul Ricœur, La Mémoire, l'histoire, l'oubli, op. cit., p. 387.

23. «Il n'y a pas d'histoire du présent, au sens strictement narratif du terme. Ce ne pourrait être qu'une anticipation de ce que des historiens futurs pourraient écrire sur nous. La symétrie entre expliquer et prédire, caractéristique des sciences nomologiques, est brisée au niveau même de l'énoncé historique. Si une telle narration du présent pouvait être écrite et être connue de nous, nous pourrions à notre tour la falsifier en faisant le contraire de ce qu'elle prédit. Nous ne savons pas, absolument pas, ce que les historiens du futur diront de nous » (Paul Ricœur, Temps et Récit, Paris, Seuil, 1983, t. I, p. 208). 
entre les deux abîmes ${ }^{24}$ ». À la lecture de ces lignes, on se rend compte que le présentisme tel qu'il est pratiqué par la critique shakespearienne actuelle est différent de celui qui transparaît dans les propos de Valéry. Il faut bien convenir que le présentisme envisagé actuellement est bien plus volontaire : son amnésie du passé est affirmée, et son repli dans l'autoréférentialité est revendiqué pour des raisons idéologiques ${ }^{25}$. Hawkes, Grady et Fernie (pour ne mentionner que les figures les plus revendicatives du présentisme) nous parlent d'un présent qui ne serait déterminé que par lui-même, c'està-dire en fait par la subjectivité de ses interprètes. Mais que ne ferait-on pas dire au présent au nom du présentisme? Si toutes les vérités sont relatives, subjectives, qui, parmi toutes ces voix émanant du présent, doit-on croire alors? Nous touchons là aux limites d'un courant critique qui pourrait bien être la première victime de son subjectivisme poussé à l'extrême.

Le présentisme qu'une partie de la critique shakespearienne actuelle tente d'ériger en école de pensée est également différent du présentisme des Élisabéthains, qui apparaît essentiellement comme une modalité du scepticisme chrétien. Seul Dieu peut prétendre à un point de vue véritablement englobant et à une pensée totalisante. De fait, pour les sceptiques, à l'instar de Cornelius Agrippa, dont le De incertitudine et vanitate scientiarum parut dans sa traduction anglaise en 1575, toutes les sciences humaines sont en partie marquées au sceau de l'imperfection; elles sont le résultat d'un consensus lui-même issu de la tradition, et elles se basent pour beaucoup d'entre elles sur des hypothèses ${ }^{26}$. Seule la foi du chrétien peut être source de certitude, et il vaut mieux donc s'avouer ignorant que de pécher par orgueil (p. 183). Dans une veine similaire, Philip Sidney montrait combien l'histoire reposait encore à ses yeux sur des fondements épistémologiques douteux. Pour lui, l'histoire demeurait surtout une rhétorique qui cherchait à se faire passer pour vraie en

24. Paul Valéry, « La crise de l'esprit » [1919], Euvres, éd. Jean Hytier, Paris, Gallimard, 1957, p. 988-994; p. 993. Valéry reprendra ce thème présentiste en 1935 : « D’un côté, un passé qui n'est pas aboli ni oublié, mais un passé duquel nous ne pouvons à peu près rien tirer qui nous oriente dans le présent et nous donne à imaginer le futur. De l'autre, un avenir sans la moindre figure » (« Le bilan de l'intelligence », ibid., p. 1058-1083; p. 1063).

25. Fredric Jameson parle même d'une "pathologie distinctement auto-réferentielle » à propos du postmodernisme (Postmodernism, or, the Cultural Logic of Late Capitalism, Londres, Verso, 1991, p. 12). François Hartog a lui aussi très bien identifié ce phénomène de repli présentiste, de même que ses contradictions : « le présent, au moment même où il se fait, désire se regarder comme déjà historique, comme déjà passé. Il se retourne en quelque sorte sur lui-même pour anticiper le regard qu'on portera sur lui, quand il sera complètement passé, comme s'il voulait "prévoir" le passé, se faire passé avant même d'être encore pleinement advenu comme présent; mais ce regard, c'est le sien, à lui présent. Cette tendance à transmuer le futur en futur antérieur peut aller jusqu'à la caricature » (Régimes d'historicité : Présentisme et expériences du temps, Paris, Seuil, 2003, p. 127).

26. Henrie Cornelius Agrippa, Of the Vanitie and uncertaintie of Artes and Sciences, Londres, Henrie Bynneman, 1575, p. 5. S.T.C. : 205. Références subséquentes données dans le texte. 
dissimulant le fait que, comme tout savoir humain, elle s'avérait limitée : « en affirmant ces nombreuses choses, l'historien a peine à ne pas proférer de nombreux mensonges, car le savoir humain demeure incertain ${ }^{27}$. » Dans L'histoire du monde (1614), Ralegh accordait quant à lui une large place à l'histoire biblique, soulignant combien l'homme était ignorant des causes mêmes de son existence. Le présentisme de Ralegh était teinté de scepticisme chrétien, mais aussi du pessimisme d'un homme que l'existence n'avait pas épargné. De sa prison, il écrivait «Car tout ce qui est derrière nous n'est plus rien, et tout ce qui est à venir n'est qu'un espoir trompeur $^{28} »$.

Soucieux d'octroyer au théâtre une caution morale, Thomas Heywood déclarait que les dramaturges détournaient volontairement le passé pour l'édification de leurs contemporains. Le passé, servant de toile de fond, devenait alors un pur prétexte pour parler du présent :

Quand nous racontons une histoire qui se passe ailleurs, l'intrigue est ainsi faite qu'en narrant la vie des Romains, des Grecs, ou d'autres peuples, ce sont en réalité nos compatriotes dont nous vantons les mérites ou dont nous dénonçons les vices ${ }^{29}$.

Il est certain que le théâtre élisabéthain, comme tout théâtre, avait un pouvoir d'intervention dans le présent, pouvoir qu'il revendiquait parfois ouvertement. Hamlet ne confiait-il pas à Polonius qu'il fallait prendre soin des comédiens, car ceux-ci avaient le pouvoir de faire et défaire les réputations : " qu'ils soient bien traités, car ils sont les abrégés, les brèves chroniques de notre temps ${ }^{30}$. $)$ Il reste que le théâtre de Shakespeare nouait une relation bien plus complexe avec le passé que ne peut le laisser entendre le commentaire de Heywood. Shakespeare comme Jonson, nous le savons, contemplaient aussi d'un œil critique les procédés de détournement du passé au profit du présent, mais aussi les efforts de certains de leurs contemporains pour commercialiser un présent de pacotille.

27. Philip Sidney, A Defence of Poetry, éd. J. A. Van Dorsten, Oxford, Oxford University Press, 1966, p. 52. Ma traduction.

28. Walter Ralegh, The History of the World, éd. C. A. Patrides, Londres, Macmillan, 1971, p. 69. Ma traduction.

29. Thomas Heywood, An Apology for Actors [1612], intro. et notes J. W. Binns, New York, Johnson Reprint, 1972, sig. F3². Ma traduction.

30. William Shakespeare, Hamlet : l'histoire tragique d'Hamlet prince de Danemark, trad. André Markowicz, préf. Margaret Jones-Davies, Besançon, les Solitaires intempestifs, 2009, p. 112 (Acte II, scène II). Voir aussi plus loin (p. 115), un passage dans lequel Hamlet attribue au théâtre le pouvoir de démasquer les coupables. Ralegh mettait lui en garde ses lecteurs contre les conséquences d'un présentisme trop appuyé. À ceux parmi eux qui auraient été curieux de savoir pourquoi il n'avait pas écrit l'histoire de son époque, Ralegh répondait qu'il y avait danger : " qui talonnera de trop près la vérité en écrivant l'histoire contemporaine pourra y perdre ses dents » (Walter Ralegh, op. cit., p. 80; ma traduction). 
Il ne faut pas non plus prendre le scepticisme chrétien au pied de la lettre, mais considérer que s'il insistait bien sur le caractère limité de la connaissance humaine, il contenait aussi une critique implicite des méthodes scientifiques en vigueur, et qu'il eut par conséquent un effet qui peut paraître un tant soit peu inattendu à nos yeux : il contribua, à sa façon, à l'avancée de la science par la critique de ses méthodes. Ainsi Agrippa ciblait-il les historiens, dénonçant leur détournement de l'histoire pour des raisons politiques, ou de vengeance personnelle, mais aussi pour consolider tous les pseudo-mythes nationaux. Ces historiens ne sont que des flagorneurs corrompus, écrivait en substance Agrippa, qui n'hésitent pas à mêler récits fabuleux et réalité historique pour les besoins de leur cause (ibid., p. 15-16).

Samuel Daniel, le poète-historien, avait conscience des déformations que les historiens faisaient subir au passé, et soulignait la condescendance avec laquelle le présent traitait parfois le passé :

Pardonne-nous, Antiquité, si nous dénonçons à tort tes actions, qui (comme les hommes) sont toujours soumises aux modes et à l'influence du temps, et restent tributaires de l'opinion que peut en avoir le présent. Nous te traitons comme la postérité nous traitera (celle-ci s'imaginant toujours plus sage) : elle jugera de nos erreurs selon son humeur ${ }^{31}$.

Ces critiques, soulignant la trop grande part de subjectivité des récits historiques, montrent que dans les milieux littéraires un nombre non négligeable d'auteurs avaient engagé une réflexion sur les méthodes de l'histoire et que, si les dramaturges, les pamphlétaires et les poètes soulignaient les déficiences des chroniqueurs, ce n'était pas seulement parce qu'ils souhaitaient abattre la concurrence, mais aussi sans doute parce qu'une histoire partisane, une histoire dévoyée et parfois manipulatrice, ne faisait pas honneur non plus à l'idée d'un passé envers lequel le présent reconnaissait volontiers qu'il avait une dette. Chez les historiens, des changements significatifs de méthode avaient lieu, et s'il est exagéré de parler de "révolution historique», il ne faudrait pas non plus prendre les contemporains de Shakespeare pour des naïfs. Les Elisabéthains ne sont pas nos contemporains, bien que certains de leurs problèmes épistémologiques nous affectent encore, à l'heure où le statut de l'histoire et plus généralement la validité des sciences humaines sont encore des questions qui se posent. Le présentisme de certains des contemporains de Shakespeare n'est pas non plus le nôtre. Il est empreint de doutes, mais son scepticisme trahit aussi souvent (mais pas systématiquement) le désir paradoxal d'établir la connaissance humaine sur des bases plus solides. Chez les meilleurs historiens de l'époque, on peut percevoir également la volonté de trouver un juste équilibre entre une recherche dont l'objet est le 1621, p. 101. S.T.C. : 6250 . Ma traduction. 
passé, et les nécessités d'un présent qui a besoin du passé pour s'appréhender et se construire. Dans son Histoire des dîmes (1618), John Selden évoque justement les dangers d'une recherche centrée de manière excessive sur le passé au point de devenir stérile, ou, à l'inverse, d'un intérêt trop prononcé pour le présent conduisant à une amnésie du passé ${ }^{32}$.

\section{Vers une conception dialectique de la temporalité}

Poussé à l'extrême, le présentisme actuel transforme nos représentations du temps : nous voyons lentement se dessiner devant nos yeux l'image d'un vaste présent qui phagocyte le passé (lequel ne peut plus être conçu que sous les traits du présent), et s'approprie également l'avenir qui n'est plus qu'une extension de ses préoccupations et de ses prévisions. Créature tentaculaire, le présentisme a des aspirations totalisantes malgré son subjectivisme souvent radical. Mais son travail d'appropriation du passé tend à gommer la dialectique implicite entre le passé et le présent, qui est à la base de ce qui constitue la « modernité », telle que nous l'avons définie plus haut.

Le présentisme ne conçoit pas le passé comme Autre : l'altérité du passé est dénoncée par lui comme une notion peu fructueuse (pour ne pas dire stérile), car elle détourne des enjeux du présent. Le présentisme préfère ne voir dans le passé que la réflexion de ses propres traits. C'est donc sous le visage du Même que le présentisme se plaît à envisager le passé. Ce faisant, il ignore, ou tente de court-circuiter, la dialectique par laquelle se constitue notre idée du passé, mais aussi notre conception de la «modernité ». Cette dialectique implique que notre relation au passé est en réalité dynamique, que nous considérons le passé tantôt comme Autre, tantôt comme Même, et tantôt sur un mode de simple analogie. De fait, nous construisons notre rapport au passé (et par conséquent notre conception du présent) par le biais de processus de familiarisation et de défamiliarisation, d'identification et de distanciation, qui peuvent s'exercer à des degrés divers et sans que l'un soit nécessairement contradictoire avec l'autre. En conséquence, il n'est pas impensable de prétendre que le passé reste intelligible parce qu'il persiste encore dans le présent (identification), et cette position n'est pas obligatoirement incompatible avec la proposition selon laquelle le passé est un Autre qui permet de faire entendre la différence du présent (distanciation). La dialectique se brise, en revanche, lorsque la distance temporelle est ignorée totalement ou que celle-ci est considérée comme hors de propos (cas du présentisme). À l'inverse, la dialectique peut également s'interrompre lorsque la prise de distance est telle que celle-ci

32. John Selden, The Historie of Tithes That Is, the Practice of Payment of Them. The Positiue Laws Made for Them. The Opinions Touching the Right of Them. A Review of It. Is Also Annext, Which Both Confirmes It and Directs in the Vse of It. By I. Selden, Londres, s. n., 1618, sig. a2 ${ }^{\mathrm{rv}}$. STC : 22172.3. 
empêche toute empathie, toute "re-familiarisation du non-familier ${ }^{33}$ ». Ce qui est important, on l'aura compris, c'est la relation qui s'établit entre le passé vécu par les êtres humains d'autrefois et nous. C'est le travail de l'histoire de mettre au jour cette relation, qui doit être sans cesse renouvelée, mais ce peut être aussi le rôle du théâtre, notamment lorsque son objet est d'explorer la relation des êtres humains au temps et à l'histoire.

Jean-Christophe Mayer IRCL (UMR 5186) - Université Paul Valéry-Montpellier III

33. Paul Ricœur, Temps et récit, t. III, p. 213. Sur le Même, l'Autre et l'Analogue, voir ibid., p. 205-206, 226. 